\title{
Determination of Pesticides Residues in Eggplant and Tomatoes from Central Marked in Khartoum State Using Quechers Method and Gas Liquid Chromatography-Mass Spectrometry
}

\author{
Salah Eldeen A Ali ${ }^{1,2 *}$, Mohamed E Abdel Aziz ${ }^{3}$ and Salah E Mohamed ${ }^{1}$ \\ ${ }^{1}$ University of Khartoum, Faculty of Science, Chemistry Department, Sudan \\ ${ }^{2}$ National Chemical Laboratories, National Public Health Laboratory, Federal Ministry of Health, Sudan \\ ${ }^{3}$ University of Sudan, Faculty of Science, Chemistry Department, Sudan \\ *Corresponding author: Salah Eldeen A Ali, University of Khartoum, Faculty of Science, Chemistry Department, Khartoum, Sudan
}

\section{ARTICLE INFO}

Received: 㗀 December 18, 2019

Published: 幽January 07, 2020

Citation: Salah Eldeen A Ali, Mohamed E Abdel Aziz, Salah E Mohamed. Determination of Pesticides Residues in Eggplant and Tomatoes from Central Marked in Khartoum State Using Quechers Method and Gas Liquid Chromatography-Mass Spectrometry. Biomed J Sci \& Tech Res 24(2)2020. BJSTR. MS.ID.004035.

Abbreviations: MRL: Maximum Residue Limits; GC: Gas Chromatography; ECD: Electron Capture Detectors; MS: Mass Spectrometry; SIM: Selected Ion Monitoring; EC: European Commission; RSD: Relative Standard Deviation; LOD: Limit of Detection; LOQ: Limit of Quantification

\begin{abstract}
Pesticides are widely used in food production to increase food security, but they can have negative health effects on consumers. Both raw and processed vegetables have been found to be contaminated with pesticide residues. Consumers can be exposure to pesticides via food consumption. In this paper, we present the investigation of 30 pesticides in total of 90 samples of tomatoes and 27 samples of eggplant from central markets in Khartoum state using the quick easy cheap effective rugged and safe (QuEChERS) multi-residue extraction, followed by gas chromatography-mass spectrometry (GC-MS). Pesticide residues were not detected in 9 samples (8\%), while 108 samples (92\%) contained detectable amount of pesticide residues. The percentage of contaminated vegetable samples was high for both tomatoes (91.2\%) and eggplant (96.3\%). Multiple pesticides residues were detected on 65samples with percentage of $(55.6 \%)$ from total analyzed samples. Multiple residues with four pesticides were present in two samples of tomatoes. Sixteen pesticides chloropyrifos, diazinon, cypermethrin, deltamethrin, omethoate, lamdacyhalothrin, dimethoate, fenvlarate, fenpropathrin, pendimethalin, endosulfan, imidacloprid, carbaryl, permethrin, malathion and profenofos were detected in the analyzed vegetable samples with concentration range between 0.008-1.882 $\mathrm{mg} / \mathrm{kg}$ and mean range between $0.0225-0.8631 \mathrm{mg} / \mathrm{kg}$. Summer and autumn were the most seasons contaminated with pesticides residues. It is concluded that the continuous monitoring and strict regulation of pesticide use on food crops, especially vegetables, are necessary.
\end{abstract}

Keywords: Pesticide residues; Tomatoes; Eggplant; QuEChERS; GC MS; Seasons; Markets; Khartoum

\section{Introduction}

A pesticide defines as "any substance or mixture of substances intended for preventing, destroying, attracting, repelling, or controlling any pest including unwanted species of plants or animals during the production, storage, transport, distribution, and processing of food, agricultural commodities, or animal feeds or which may be administered to animals for the control of ectoparasites, pesticides are part of a large group of organic compounds that present extremely diverse physico-chemical properties and are widely used in the control or prevention of diseases in various crops, such as fruits, vegetables and cereals $[1,2]$. The use of pesticides in agriculture has helped to increase productivity and rising food production, most pesticides are 
applied directly to the soil or sprayed over crop fields and hence are release directly to the environment. However, the continued use of pesticides specially insecticides increases the possibility of residues to be found in some vegetables, threatening the alimentary security. Pesticide residue refers to the pesticides that may remain on or in food after they are applied to food crops [3]. Pesticide residues in agriculture have been a crucial worldwide problem that directly threatened the environment and people's health. Over 1000 compounds may be applied to agricultural crops in order to control undesirable moulds, insects or weeds to ensure the safety of food for consumers, numerous legislations such as codex directives and European Commission have established Maximum Residue Limits (MRLs) for pesticides in foodstuffs [4,5].

The determination of pesticide residues in agricultural products, plant and environmental samples has been a major subject for many years because of their toxic potential risk for human health, persistence and tendency to bioaccumulate. For many years, analysis of fruit and vegetable samples for organophosphorus, organochlorine, and pyrethroids pesticides at low levels were by gas chromatography (GC) using selective detectors: flame photometric, nitrogen phosphorus, and electron capture detectors (FPD, NPD, and ECD). Although these detectors are sufficiently sensitive for compliance with maximum residues limits (MRLs) in European Union regulations, they provide poor specificity for confirmation in these matrixes; therefore, GC coupled with mass spectrometry (MS) was required [6,7]. Many investigations studies form many areas were reported that different type of pesticides left residual amounts in fruit and vegetables with residues levels in rang of $\mathrm{mg} / \mathrm{kg}$ [8-10], also many methods and studies have reported the use of GC/MS and LC/MS with either full scan or selected ion monitoring (SIM) to control pesticide residues in matrixes such as fruit, vegetables, milk, and soils $[11,12]$. During the last years, the Quick, Easy, Effective, Cheap, Rugged, and Safe (QuEChERS) approach, has become very popular for the determination of pesticides on diverse food matrices include fruits and vegetables, this method has been readily accepted by many pesticide residue analysts because of its low organic solvent consumption, low cost per sample, fast, the accurate procedures non time consuming, and have high analyte recoveries [13,14]. Vegetables are commonly used to fulfill the balance diet requirement as they are a vital source of vitamins and fiber, tomato (Lycopersicon esculentum) and eggplant (Solanum melongena) are from the most popular and widely grown vegetables in the world, tomato and eggplant crops are much susceptible to insect attack and disease infestation from seedling to fruiting stage. Eggplant helps to prevent colon cancer, reduces cholesterol level, helps in the type 2 diabetes management, is very rich in antioxidant, and helps to control weight $[15,16]$.

\section{Materials and Methods}

\section{Study Area and Time}

The tomatoes and eggplants samples were collected from central markets of Khartoum state including Khartoum central market in Khartoum, Khartoum north central market in Khartoum north and Omdurman market (elshaabi) in Omdurman during summer, winter and autumn seasons during 2017.

\section{Sample Collection and Treatment}

A total of 117 vegetables samples were collected for pesticide residue analysis. The vegetable samples included 90 samples of tomatoes and 27 samples of eggplants. The sampling was performed in accordance with the general principles and methods of the European Commission (EC) directive 2002/63/EC [17]. Each representative vegetable sample was a composite of 6 to 10 subsamples of the same commodity collected through random sampling. All the samples (1-2 kg each) were placed in polythene bags, in box contain ice, to avoid contamination and deterioration, labeled, and transported to the laboratory and stored at $-20^{\circ} \mathrm{C}$ until analysis.

\section{Chemicals and Reagents}

Pesticide reference standards including malathion, diazinon, profenofos, chlorpyrifos, chlorpyrifos-methyl, omethoate, dimethoate, cypermethrin, deltamethrin, fenpropathrin, permethrin, tetramethrin, cyfluthrin, fenvlarate, lamdacyhalothrin , lindane, heptachlor, aldrin, heptachlor epoxide, mixture endosulfan, dieldrin, endrin, o,P-DDT, carbaryl, carbofuran, bendiocarb, atrazine, imidacloprid, thiomethoxam and pendimethalin were purchased from Sigma Aldrich GmbH (Augsburg, Germany), Supelco and Bayer Crop Science, with certified purity ranging from $95 \%$ to $99 \%$. Acetonitrile, acetone, sodium chloride, toluene, acetic acid and anhydrous magnesium sulfate were obtained from Scharlau (Barcelona, Spain). QuEChERS Finisterre micro centrifuge tube containing two or more of primary secondary amine (PSA), anhydrous magnesium sulfate (MgSO4) and graphed carbon black (GCB) with details of TR-Q2035 (150mg MgSO4, 25mg PSA, 2.5mg GCB) and TR-Q2015 (150mg MgSO4, 25mg PSA) were obtained from Teknokroma (Barcelona, Spain). Piperonyl Butoxide (PPB) with certified purity $95 \%$ used as internal standard was obtained from Sigma Aldrich $\mathrm{GmbH}$. All the organic solvents used were high performance liquid chromatography (HPLC) grade.

\section{Preparation of Pesticide Standard Solution}

Pesticide standard stock solutions (1000 mg/L) of malathion, diazinon, profenofos, chlorpyrifos, chlorpyrifos-methyl, omethoate, dimethoate, cypermethrin, deltamethrin, fenpropathrin, permethrin, tetramethrin, cyfluthrin, fenvlarate, lamdacyhalothrin , lindane, heptachlor, aldrin, heptachlor epoxide, mixture endosulfan, dieldrin, endrin, o,P-DDT, carbaryl, carbofuran, bendiocarb, atrazine, imidacloprid, thiomethoxam and pendimethalin were prepared separately with acetone and stored at $-20{ }^{\circ} \mathrm{C}$ until use. A mixed pesticide standard solution of $50 \mathrm{mg} / \mathrm{L}$ was prepared by adding the proper volume of each individual stock solution and diluted to volume. Intermediate mixed pesticide standard solution of $10 \mathrm{mg} / \mathrm{L}$ was prepared from the mixed pesticide standard solution of $50 \mathrm{mg} / \mathrm{L}$. Matrix-matched calibration standards were prepared by adding mixed pesticide standard working solutions in the blank 
extract to reach the final concentrations of $0.005,0.01,0.05,0.10$, 0.20 and $0.50 \mathrm{mg} / \mathrm{kg}$. Calibration standards in acetone were also prepared at the same concentration levels. All the standards were kept in a freezer at $-20^{\circ} \mathrm{C}$ until use.

\section{Sample Extraction and Cleanup Procedure}

Ten grams of properly homogenized vegetable sample was taken in a 50-mL screw-capped polypropylene centrifuge tube, and $10 \mathrm{ml}$ acetonitrile (MeCN) contain 1\% acetic acid was added into the centrifuge tube. The centrifuge tube was closed properly and shaken vigorously for $30 \mathrm{~s}$ by vortex mixer. Then, $4 \mathrm{~g}$ anhydrous MgSO4, 1g sodium chloride, $1 \mathrm{~g}$ trisodium citrated dehydrate and $0.5 \mathrm{~g}$ disodium hydrogen citrated sesquihydrate were added into the centrifuge tube, and it was shaken by vortex mixer for 1 min. afterward, the extract was centrifuged for $5 \mathrm{~min}$ at 5,000 rpm. An aliquot of $1 \mathrm{~mL}$ of the MeCN layer was transferred into a micro centrifuge tube containing 150mg anhydrous MgSO4, 25mg PSA, $2.5 \mathrm{mg}$ GCB for tomatoes or containing $150 \mathrm{mg}$ anhydrous MgSO4 and 25mg PSA for eggplants. The content of the centrifuge tube was thoroughly mixed by vortex for $30 \mathrm{~s}$ and centrifuged for $5 \mathrm{~min}$ at $4,000 \mathrm{rpm}$. A one mL supernatant was taken into a clean test tube, and one drop of toluene was added into the test tube. The test tube containing the extract was stored at $-20{ }^{\circ} \mathrm{C}$ until analysis.

\section{Gas Chromatography-Mass Spectrometry Analyses}

A Shimadzu (QP 2010 GC-MS) gas chromatography equipped with mass selective detector and a RTX-5MS column (30 m long, $0.25 \mathrm{~mm}$ internal diameter, and $0.25 \mathrm{~nm}$ film thickness) was used for analysis. Sample injection was performed in the split less mode, with an injector temperature of $250{ }^{\circ} \mathrm{C}$. The temperature of the was programmed from an initial value of $600 \mathrm{C}$ for $2 \mathrm{~min}$, ramped to $150 \mathrm{OC}$ at $10 \mathrm{OC} / \mathrm{min}$ for $10 \mathrm{~min}$, and to $250 \mathrm{OC}$ at $20 \mathrm{OC} / \mathrm{min}$ for $10 \mathrm{~min}$, and was raised to $270 \mathrm{OC}$ at $10 \mathrm{OC} / \mathrm{min}$ for $15 \mathrm{~min}$. Helium (99.999\% purity) was used as a carrier gas with a constant flow rate of $1 \mathrm{~mL} / \mathrm{min}$. For the mass detector MS, the ion source and interface temperatures were maintained at 220 and $2700 \mathrm{C}$ respectively. Electron ionization (EI) was used at $70 \mathrm{eV}$ in selective ion monitoring (SIM) and full-scan modes between $50 \mathrm{~m} / \mathrm{z}$ and 500 $\mathrm{m} / \mathrm{z}$ for the detection of different analytes. The solvent cut and total run time were 5 and $53 \mathrm{~min}$, respectively.

\section{Quality Control}

The performance of the QuEChERS method was evaluated by performing recovery studies. The recovery rate and precision of the method (expressed as relative standard deviation (RSD), \%) were measured by analyzing replicate pesticide-free samples of each type of vegetable, which were fortified at a concentration of 0.01 or 0.05 $\mathrm{mg} / \mathrm{kg}$ for each pesticide. Sensitivity was evaluated by determining the limit of detection (LOD) and limit of quantification (LOQ), using the signal-to-noise ratio $(\mathrm{S} / \mathrm{N})$ of 3:1 and10:1, respectively [18].

\section{Results and Discussion}

\section{Pesticide Residues and Multiple Residues in Analyzed Samples}

The level of pesticide residues in 117 vegetable samples was determined. Pesticide residues were not detected in 9 samples (8\%), while 108 samples (92\%) contained detectable amount of pesticide residues. The percentage of contaminated vegetable samples was high for both tomatoes (91.2\%) and eggplant (96.3\%); eggplant has the highest percentage of contaminated samples. No pesticide residues were detected in only one sample of eggplant and 8 samples of tomatoes Figure 1. Multiple pesticides residues were detected on 65samples with percentage of (55.6\%) from total analyzed samples; in tomatoes from 90 samples analyzes only 51(56.6\%) samples contain multiple pesticides resides from them 31 (34.3\%), 18 (20\%) and $2(2.2 \%)$ samples contain two, three and four kind of pesticides resides respectively. In eggplant from 27 samples analyzes only 14 (51.9\%) samples contain multiple pesticides resides from them 12 (44.4\%) and 2 (7.4\%) samples contain two and three kind of pesticides resides respectively Figure 2.

\section{With pesticide residues $\quad$ With out pesticide residues}

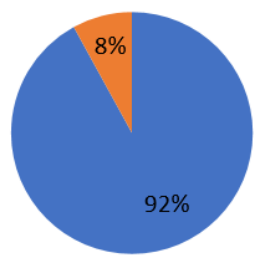

Figure 1: Percentages of samples without pesticides residue and with pesticides residue from the total number of samples analyzed.

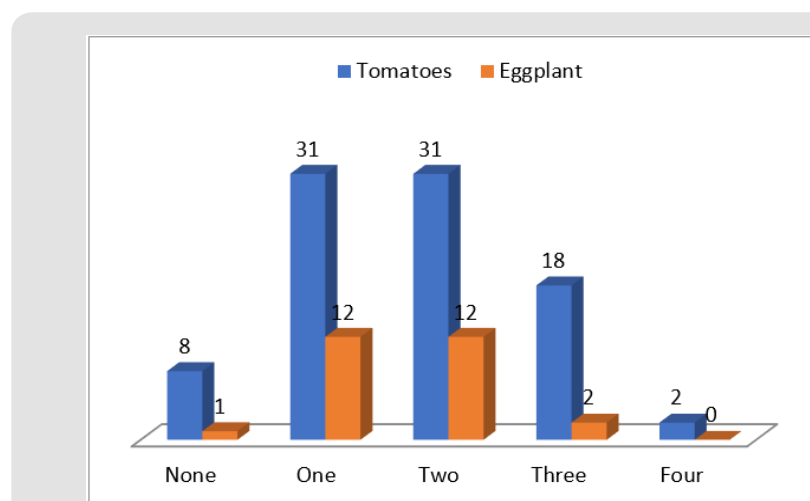

Figure 1: Number of different vegetable samples contains one or more pesticides residues.

\section{Detection Frequencies of Pesticides in Analyzed Samples}

From the 30 pesticides studied, only 16 pesticides were detected in the analyzed vegetable samples. Permethrin has been the most 
common pesticides detected in the analyzed vegetable samples (21 samples) with concentration range between $0.032-1.025 \mathrm{mg} /$ $\mathrm{kg}$ followed by Endosulfan (20 samples) with concentration range between $0.015-0.423 \mathrm{mg} / \mathrm{kg}$. Carbaryl has been detected in17 samples with concentration range between 0.021- 1.052 $\mathrm{mg} / \mathrm{kg}$. Sixteen vegetable samples were contaminated by each of Cypermethrin, Deltamethrin and Malathion with concentration range of (0.008 - 0.051), (0.011 - 1.322) and (0.019-0.251) mg/kg respectively. Diazinon has been detected in fourteen samples with concentration range of $(0.114-0.543) \mathrm{mg} / \mathrm{kg}$. Thirteen samples were contaminated by each of Profenofos and Fenpropathrin with concentration range of $(0.212-1.882)$ and $(0.025-0.311)$ $\mathrm{mg} / \mathrm{kg}$ respectively. Chloropyrifos has been detected in eleven samples with concentration range of (0.035-0.123) $\mathrm{mg} / \mathrm{kg}$. Lamdacyhalothrin, Dimethoate, Pendamethalin, Fenvlarate, Imidcloprid and Omethoate have been detected in 9, 8, 8, 7, 6 and 4 samples respectively Table1.

Table 1: Means and ranges of pesticides residues found in analyzed vegetable samples.

\begin{tabular}{|c|c|c|c|c|c|c|c|c|}
\hline \multirow{2}{*}{ Pesticides } & \multicolumn{3}{|c|}{ Sample Information } & \multirow{2}{*}{ Range $\mathrm{mg} / \mathrm{kg}$} & \multirow{2}{*}{$\begin{array}{c}\text { Mean of (+) } \\
\text { samples }\end{array}$} & \multirow{2}{*}{$\begin{array}{l}\text { Mean of total } \\
\text { samples }\end{array}$} & \multirow{2}{*}{ Std D } & \multirow{2}{*}{$\begin{array}{l}\text { Mean Std } \\
\text {.Error }\end{array}$} \\
\hline & Total No & No of $(+)$ & $\%$ of $(+)$ & & & & & \\
\hline Carbaryl & 117 & 17 & 14.5 & $0.022-1.052$ & 0.4117 & 0.0637 & 0.2039 & 0.0189 \\
\hline Chloropyrifos & 117 & 11 & 9.4 & $0.035-0.123$ & 0.0664 & 0.0062 & 0.0214 & 0.0019 \\
\hline Cypermethrin & 117 & 16 & 13.7 & $0.008-0.051$ & 0.0225 & 0.0035 & 0.0101 & 0.0009 \\
\hline Deltamethrin & 117 & 16 & 13.7 & $0.011-1.322$ & 0.5946 & 0.0813 & 0.2039 & 0.0225 \\
\hline Diazinon & 117 & 14 & 12 & $0.114-0.543$ & 0.2961 & 0.0354 & 0.1074 & 0.0099 \\
\hline Dimethoate & 117 & 8 & 6.8 & $0.042-0.313$ & 0.2188 & 0.0124 & 0.0515 & 0.0048 \\
\hline Endosulfan & 117 & 20 & 17.1 & $0.015-0.423$ & 0.1623 & 0.0286 & 0.0789 & 0.0073 \\
\hline Fenpropathrin & 117 & 13 & 11.1 & $0.025-0.311$ & 0.1149 & 0.0128 & 0.0457 & 0.0042 \\
\hline Fenvlarate & 117 & 7 & 6 & $0.073-0.253$ & 0.1496 & 0.0089 & 0.0388 & 0.0036 \\
\hline Imidcloprid & 117 & 6 & 5.1 & $0.051-0.211$ & 0.1312 & 0.0067 & 0.0322 & 0.0029 \\
\hline Lamdacyhalothrin & 117 & 9 & 7.7 & $0.034-0.212$ & 0.0952 & 0.0073 & 0.0298 & 0.0028 \\
\hline Malathion & 117 & 16 & 13.7 & $0.019-0.251$ & 0.1243 & 0.017 & 0.0521 & 0.0048 \\
\hline Omethoate & 117 & 4 & 3.4 & $0.113-0.332$ & 0.2045 & 0.0069 & 0.041 & 0.0038 \\
\hline Pendamethalin & 117 & 8 & 6.8 & $0.123-0.424$ & 0.2014 & 0.0139 & 0.0569 & 0.0053 \\
\hline Permethrin & 117 & 21 & 17.9 & $0.032-1.025$ & 0.3466 & 0.0671 & 0.02 & 0.0185 \\
\hline Profenofos & 117 & 13 & 11.1 & $0.212-1.882$ & 0.8631 & 0.0959 & 0.3235 & 0.0299 \\
\hline
\end{tabular}

Pesticides residues in different Markets and Seasons in Analyzed Samples

In eggplant only five samples (18.5\%) contain pesticides residues more than codex maximum residues limits, carbaryl has been detected in nine samples( 33.3\%) from each seasons and markets and only one sample from khartoum north market in autumn has been found above the codex maximum residues limits cypermethrin has been detected in six samples(22.2\%)and only one sample from omdurman market in summer has been found above the codex maximum residues limits, endosulfan has been detected in five samples(18.8\%)and only two sample from khartoum central and omdurman in summer and autumn respectively have been found above the codex maximum residues limits, imidacloprid has been detected in six samples(22.2\%) and only one sample from omdurman market in summer has been found above the codex maximum residues limits. Each permethrin and fenpropathrin has been detected in 7 (25.9\%) and 8 (29.6\%) samples respectively but no sample contains permethrin above the codex maximum residues limits Table 2. 
Table 2: Comparison between markets and seasons in pesticides residues found in Eggplant. S (Summer), A (Autumn), W (Winter) and - (no MRLs established by Codex).

\begin{tabular}{|c|c|c|c|c|c|c|c|c|c|c|c|}
\hline \multirow{2}{*}{ Pesticides } & \multicolumn{3}{|c|}{ Khartoum central market } & \multicolumn{3}{|c|}{ Khartoum north } & \multicolumn{3}{|c|}{ Omdurman } & \multirow{2}{*}{ No above MRL } & \multirow{2}{*}{ CODEX MRL [19] } \\
\hline & $\mathbf{S}$ & $\mathbf{A}$ & w & S & $\mathbf{A}$ & $\mathbf{w}$ & S & A & $\mathbf{w}$ & & \\
\hline Carbaryl & $\begin{array}{c}1 \\
0.953\end{array}$ & $\begin{array}{c}1 \\
0.882\end{array}$ & $\begin{array}{c}1 \\
0.521\end{array}$ & $\begin{array}{c}1 \\
0.072\end{array}$ & $\begin{array}{c}1 \\
1.052\end{array}$ & $\begin{array}{c}1 \\
0.062\end{array}$ & $\begin{array}{c}1 \\
0.822\end{array}$ & $\begin{array}{c}1 \\
0.759\end{array}$ & $\begin{array}{c}1 \\
0.052\end{array}$ & 1 & 1 \\
\hline Cypermethrin & $\begin{array}{c}1 \\
0.029\end{array}$ & $\begin{array}{c}1 \\
0.015\end{array}$ & 0 & $\begin{array}{c}1 \\
0.019\end{array}$ & 0 & $\begin{array}{c}1 \\
0.008\end{array}$ & $\begin{array}{c}1 \\
0.045\end{array}$ & 0 & $\begin{array}{c}1 \\
0.008\end{array}$ & 1 & 0.03 \\
\hline Endosulfan & $\begin{array}{c}1 \\
0.052\end{array}$ & $\begin{array}{c}1 \\
0.095 \\
\end{array}$ & $\begin{array}{c}1 \\
0.105\end{array}$ & 0 & 0 & 0 & $\begin{array}{c}1 \\
0.095\end{array}$ & $\begin{array}{c}1 \\
0.125 \\
\end{array}$ & 0 & 2 & 0.1 \\
\hline Fenpropathrin & $\begin{array}{c}1 \\
0.112\end{array}$ & $\begin{array}{c}1 \\
0.209\end{array}$ & 0 & $\begin{array}{c}1 \\
0.172\end{array}$ & $\begin{array}{c}1 \\
0.311\end{array}$ & $\begin{array}{c}1 \\
0.025\end{array}$ & $\begin{array}{c}1 \\
0.185\end{array}$ & $\begin{array}{c}1 \\
0.089\end{array}$ & $\begin{array}{c}1 \\
0.052\end{array}$ & - & - \\
\hline Permethrin & $\begin{array}{c}1 \\
0.832\end{array}$ & 0 & $\begin{array}{c}1 \\
0.968\end{array}$ & $\begin{array}{c}1 \\
0.059\end{array}$ & $\begin{array}{c}1 \\
0.561\end{array}$ & 0 & $\begin{array}{c}1 \\
0.985\end{array}$ & $\begin{array}{c}1 \\
1.025\end{array}$ & $\begin{array}{c}1 \\
0.052\end{array}$ & 0 & 1 \\
\hline Imidacloprid & $\begin{array}{c}1 \\
0.162\end{array}$ & $\begin{array}{c}1 \\
0.092\end{array}$ & $\begin{array}{c}1 \\
0.051\end{array}$ & 0 & $\begin{array}{c}1 \\
0.211\end{array}$ & $\begin{array}{c}1 \\
0.076\end{array}$ & $\begin{array}{c}1 \\
0.195\end{array}$ & 0 & 0 & 1 & 0.2 \\
\hline
\end{tabular}

Pesticides residues have been detected in all tomatoes sample analyzed in summer and autumn from Khartoum central market but in winter only three samples were free of pesticides residues. The most pesticides detected in Khartoum market during the three seasons were Carbaryl, Chloropyrifos, Dimethoate, Deltamethrin, Profenofos, Lamdacyhalothrin, Malathion, Pendamethalin, Permethrin, Diazinon and Endosulfan in addition to Cypermethrin, Fenvlarate, Omethoate in summer and autumn and Fenpropathrin in autumn only. In khartoum central market only one sample in each summer and winter contain fenvlarate and deltamethrin respectively had been found more than MRLs established by codex; but in autumn three samples were been found more than MRLs established by codex contain deltamethrin in two samples and diazinon in one sample. The most pesticides detected in Khartoum north during the three seasons were Carbaryl, Dimethoate, Deltamethrin, Malathion, Pendamethalin, Permethrin, Diazinon and Endosulfan in addition to Cypermethrin, Fenvlarate, Lamdacyhalothrin, Fenpropathrin and chloropyrifos in summer and autumn and Profenofos in autumn and winter. In Khartoum north market two samples contain deltamethrin been found more than MRLs established by Codex in each summer and winter; but in autumn one sample had been found more than MRLs established by Codex contain deltamethrin. One sample from each summer and autumn contain Fenvlarate more than MRLs established by Codex [19].

The most pesticides detected in Omdurman market during the three seasons were Deltamethrin, Cypermethrin, Fenvlarate, Profenofos, Malathion, Pendamethalin, Permethrin and Endosulfan in addition to Chloropyrifos and Diazinon in summer and autumn; Carbaryl and Lamdacyhalothrin have been detected in autumn and Fenpropathrin detect in summer only. Regarding Omdurman samples deltamethrin had been found more than MRLs established by Codex in 1, 3 and 2 samples from summer, autumn and winter respectively and one sample contain fenvlarate from summer exceeded the MRLs established by Codex Table 3. This study shows the evidence of contamination of vegetables specially tomatoes and eggplant with pesticides residues in Khartoum state markets. Slightly more than $92 \%$ of the samples analyzed contained pesticides residues and from present samples more than $18.8 \%$ above MRLs established by Codex. According to results there were a little different in pesticides found in tomatoes and eggplant in the different seasons and no sample from the tow kind of vegetables contain organochlorine pesticides except Endosulfan in 15 different samples and no tomatoes samples show Endosulfan residues above MRLs set by Codex, in previous studies some vegetables from Ghana [20], Saudi Arabia [21] and China [22] contain organochlorine pesticides which were banned or restricted in majority of countries. Most of pesticides residues found in tomatoes in winter are similar to that found by Magdoleen especially for Malathion, Chloropyrifos and Dimethoate [23]. In study by Safiqul and other there were detected different organophosphorus like diazinon, malathion and chloropyrifos in eggplant from retail markets of some town in Bangladesh and there result show little different from our study specially in pesticides residues exceeded the MRLs which was dimethoate [24].In the analysis of pesticides on fresh vegetables using GC, interference and contamination resulting from plant pigments and the matrix are often encountered. Although silica gel and florisil are commonly used to reduce the matrix effects, in this study activated carbon were another material used to remove the pigments from fresh vegetables. Activated carbon is often used because it is cheap and strongly adsorbs many organic compounds in some fresh vegetables [25]. 
Table 3: Comparison between markets and seasons in pesticides residues found in Tomatoes.

\begin{tabular}{|c|c|c|c|c|c|}
\hline Market & Season & Pesticide found and amount (mg/kg)) & No(+) & No above MRL & Codex MRL [19] \\
\hline \multirow{40}{*}{ Khartoum central } & \multirow{14}{*}{ Summer } & Carbaryl $(0.022,0.252)$ & 2 & 0 & 5 \\
\hline & & Chloropyrifos $(0.055,0.064)$ & 2 & 0 & 0.5 \\
\hline & & Dimethoate (0.225. 0.313) & 2 & - & - \\
\hline & & Deltamethrin $(0.011,0.052)$ & 2 & 0 & 0.3 \\
\hline & & Cypermethrin $(0.014,0.015)$ & 2 & 0 & 0.2 \\
\hline & & Profenofos (0.452) & 1 & 0 & 10 \\
\hline & & Fenvlarate (0.154) & 1 & 1 & 0.1 \\
\hline & & Lamdacyhalothrin $(0.053,0.064,0.085)$ & 3 & - & - \\
\hline & & Malathion $(0.023,0.059)$ & 2 & 0 & 0.5 \\
\hline & & Pendamethalin (0.153) & 1 & - & - \\
\hline & & Permethrin $(0.184,0.238)$ & 2 & 0 & 1 \\
\hline & & Diazinon $(0.114,0.125,0.354)$ & 3 & 0 & 0.5 \\
\hline & & Omethoate $(0.122,0.332)$ & 2 & - & - \\
\hline & & Endosulfan $(0.113,0.192)$ & 2 & 0 & 0.5 \\
\hline & \multirow{15}{*}{ Autumn } & Carbaryl (0.451) & 1 & 0 & 5 \\
\hline & & Chloropyrifos (0.121) & 1 & 0 & 0.5 \\
\hline & & Dimethoate (0.042) & 1 & - & - \\
\hline & & Deltamethrin $(0.453,0.524)$ & 2 & 2 & 0.3 \\
\hline & & Cypermethrin $(0.024,0.042)$ & 2 & 0 & 0.2 \\
\hline & & Profenofos (0.452) & 1 & 0 & 10 \\
\hline & & Fenvlarate (0.092) & 1 & 0 & 0.1 \\
\hline & & Lamdacyhalothrin $(0.171,0.212)$ & 2 & - & - \\
\hline & & Malathion $(0.095,0.158)$ & 2 & 0 & 0.5 \\
\hline & & Pendamethalin (0.184) & 1 & - & - \\
\hline & & Permethrin (0.091) & 1 & 0 & 1 \\
\hline & & Diazinon $(0.154,0.543)$ & 2 & 1 & 0.5 \\
\hline & & Omethoate $(0.113,0.251)$ & 2 & - & - \\
\hline & & Endosulfan $(0.231,0.326)$ & 2 & 0 & 0.5 \\
\hline & & Fenpropathrin $(0.054,0.064)$ & 2 & 0 & 1 \\
\hline & \multirow{11}{*}{ Winter } & Carbaryl (0.451) & 1 & 0 & 5 \\
\hline & & Chloropyrifos (0.082) & 1 & 0 & 0.5 \\
\hline & & Dimethoate (0.212) & 1 & - & - \\
\hline & & Deltamethrin (0.519) & 1 & 1 & 0.3 \\
\hline & & Profenofos $(0.412,0.364)$ & 2 & 0 & 10 \\
\hline & & Lamdacyhalothrin (0.093) & 1 & - & - \\
\hline & & Malathion (0.064) & 1 & 0 & 0.5 \\
\hline & & Pendamethalin (0.123) & 1 & - & - \\
\hline & & Permethrin $(0.032,0.051)$ & 2 & 0 & 1 \\
\hline & & Diazinon (0.253) & 1 & 0 & 0.5 \\
\hline & & Endosulfan $(0.032,0.296)$ & 2 & 0 & 0.5 \\
\hline
\end{tabular}




\begin{tabular}{|c|c|c|c|c|c|}
\hline \multirow{36}{*}{ Khartoum north } & \multirow{13}{*}{ Summer } & Carbaryl (0.511) & 1 & 0 & 5 \\
\hline & & Chloropyrifos $(0.046,0.065)$ & 2 & 0 & 0.5 \\
\hline & & Dimethoate (0.094) & 1 & - & - \\
\hline & & Deltamethrin (0.344. 0.715) & 2 & 2 & 0.3 \\
\hline & & Cypermethrin (0.012) & 1 & 0 & 0.2 \\
\hline & & Fenvlarate $(0.215)$ & 1 & 1 & 0.1 \\
\hline & & Lamdacyhalothrin (0.054) & 1 & - & - \\
\hline & & Malathion $(0.207,0.254)$ & 2 & 0 & 0.5 \\
\hline & & Pendamethalin (0.254) & 1 & - & - \\
\hline & & Permethrin $(0.219,0.521)$ & 2 & 0 & 1 \\
\hline & & Diazinon $(0.132,0.353)$, & 2 & 0 & 0.5 \\
\hline & & Endosulfan $(0.015,0.423)$ & 2 & 0 & 0.5 \\
\hline & & Fenpropathrin (0.025) & 1 & 0 & 1 \\
\hline & \multirow{14}{*}{ Autumn } & Carbaryl (0.485) & 1 & 0 & 5 \\
\hline & & Chloropyrifos $(0.035,0.048)$ & 2 & 0 & 0.5 \\
\hline & & Dimethoate $(0.223,0.254)$ & 2 & - & - \\
\hline & & Deltamethrin (1.223) & 1 & 1 & 0.3 \\
\hline & & Cypermethrin $(0.035,0.051)$ & 2 & 0 & 0.2 \\
\hline & & Profenofos(0.212) & 1 & 0 & 10 \\
\hline & & Fenvlarate $(0.165)$ & 1 & 1 & 0.1 \\
\hline & & Lamdacyhalothrin (0.091) & 1 & - & - \\
\hline & & Malathion $(0.183,0.211)$ & 2 & 0 & 0.5 \\
\hline & & Pendamethalin (0.424) & 1 & - & - \\
\hline & & Permethrin (0.482) & 1 & 0 & 1 \\
\hline & & Diazinon $(0.254,0.452)$ & 2 & 0 & 0.5 \\
\hline & & Endosulfan (0.247) & 1 & 0 & 0.5 \\
\hline & & Fenpropathrin $(0.1544)$ & 1 & 0 & 1 \\
\hline & \multirow{9}{*}{ Winter } & Carbaryl (0.061) & 1 & 0 & 5 \\
\hline & & Dimethoate (0.087) & 1 & - & - \\
\hline & & Deltamethrin $(1.322,0.636)$ & 2 & 2 & 0.3 \\
\hline & & Profenofos $(0.676,1.245,1.882)$ & 3 & 0 & 10 \\
\hline & & Malathion $(0.025,0.052)$ & 2 & 0 & 0.5 \\
\hline & & Pendamethalin (0.195) & 1 & - & - \\
\hline & & Permethrin (0.327) & 1 & 0 & 1 \\
\hline & & Diazinon (0.394) & 1 & 0 & 0.5 \\
\hline & & Endosulfan (0.211) & 1 & 0 & 0.5 \\
\hline
\end{tabular}




\begin{tabular}{|c|c|c|c|c|c|}
\hline \multirow{30}{*}{ Omdurman } & \multirow{10}{*}{ Summer } & Chloropyrifos $(0.039,0.123)$ & 2 & 0 & 0.5 \\
\hline & & Profenofos (0.981) & 1 & 0 & 10 \\
\hline & & Deltamethrin (0.834) & 1 & 1 & 0.3 \\
\hline & & Cypermethrin (0.023) & 1 & 0 & 0.2 \\
\hline & & Fenvlarate $(0.253)$ & 1 & 1 & 0.1 \\
\hline & & Malathion $(0.214,0.251)$ & 2 & 0 & 0.5 \\
\hline & & Permethrin $(0.052,0.253)$ & 2 & 0 & 1 \\
\hline & & Diazinon $(0.231,0.294)$ & 2 & 0 & 0.5 \\
\hline & & Endosulfan $(0.045,0.351)$ & 2 & 0 & 0.5 \\
\hline & & Fenpropathrin (0.042) & 1 & 0 & 1 \\
\hline & \multirow{12}{*}{ Autumn } & Carbaryl (0.041) & 1 & 0 & 5 \\
\hline & & Chloropyrifos(0.052) & 1 & 0 & 0.5 \\
\hline & & Profenofos $(0.525,1.673)$ & 2 & 0 & 10 \\
\hline & & Deltamethrin $(0.474 .0 .576,0.952)$ & 3 & 3 & 0.3 \\
\hline & & Cypermethrin (0.043) & 1 & 0 & 0.2 \\
\hline & & Fenvlarate (0.095) & 1 & 0 & 0.1 \\
\hline & & Lamdacyhalothrin (0.034) & 1 & - & - \\
\hline & & Malathion $(0.082,0.115)$ & 2 & 0 & 0.5 \\
\hline & & Pendamethalin (0.154) & 1 & - & - \\
\hline & & Permethrin $(0.326)$ & 1 & 0 & 1 \\
\hline & & Diazinon (0.492) & 1 & 0 & 0.5 \\
\hline & & Endosulfan $(0.114,0.215)$ & 2 & 0 & 0.5 \\
\hline & \multirow{8}{*}{ Winter } & Deltamethrin $(0.921, .1 .425)$ & 2 & 2 & 0.3 \\
\hline & & Cypermethrin (0.024) & 1 & 0 & 0.2 \\
\hline & & Fenvlarate (0.073) & 1 & 0 & 0.1 \\
\hline & & Malathion (0.019) & 1 & 0 & 0.5 \\
\hline & & Pendamethalin (0.141) & 1 & - & - \\
\hline & & Permethrin $(0.072,0.521)$ & 2 & 0 & 1 \\
\hline & & Endosulfan (0.057) & 1 & 0 & 0.5 \\
\hline & & Profenofos $(0.921,1.425)$ & 2 & 0 & 10 \\
\hline
\end{tabular}

The diversity of classes of pesticide residues such as organophosphorus (Chloropyrifos, Omethoate, Dimethoate, Malathion, Profenofos and Diazinon), pyrethroids (Deltamethrin, Cypermethrin, Permethrin, Fenvlarate, Pendamethalin, Fenpropathrin and Lamdacyhalothrin) and organochlorines (Endosulfan) detected in this study show that the proposed method to determine residues of pesticides in various tomatoes and eggplant is rapid, simple, sensitive and uses smaller amount of organic solvents, reducing the risk for workers and the environment.

\section{Conclusion}

This study investigated the levels of pesticide residues in vegetable samples (tomatoes and eggplant) and compared this level between seasons and markets in Khartoum state in Sudan. The results indicated that most of the tomatoes samples were contaminated with pesticide residues, some pesticides residues concentrations in few samples of tomatoes and eggplant have been found above the MRLs established by Codex. The observed levels of pesticide residues may pose a potential health risk to consumers. Therefore, to reduce this risk, farmers should be sensitized to better pesticide safety practices and the need for continuous pesticide residue monitoring is highly recommended.

\section{References}

1. Bruzzoniti M C, Sarzanini C, Costantino G, Fungi M (2006) Determination of Herbicides by Solid Phase Extraction Gas Chromatography-Mass Spectrometry in Drinking Waters. Analytica Chimica Acta 578(2): 241249.

2. Stepán R, Tichá J, Haj slová, Kovalczuk T, Kocourek V (2005) Baby food production chain: pesticide residues in fresh apples and products. Food Additives and Contaminants 22(12): 1231-1242.

3. Bai Y, Zhou L, Wang J (2006) Organophosphorus pesticide residues in market foods in Shaanxi area, China. Food Chemistry 98(2): 240-242.

4. (2009) Food and Agricultural Organization of the United Nations. World Health Organization. 
5. (2019) European Commission. Pesticide Residue Online Database in/ on Vegetables.

6. Stephen W C Chung, Benedict L S Chen (2011) Determination of Organochlorine Pesticide Residues in Fatty Foods: A critical Review on the Analytical Methods and their Testing Capabilities. Journal of Chromatography A 1218(33): 5555-5567.

7. Guan S X, Yu Z G, Yu H N, Song C H, Song Z Q (2011) Multi-walled carbon nanotubes as matrix solid-phase dispersion extraction adsorbent for simultaneous analysis of residues of nine organophosphorus pesticides in fruit and vegetables by rapid resolution LC-MS-MS. Chromatographia 73(1): 33-41.

8. Pan J, Xia X X, Liang J (2008) Analysis of pesticide multi-residues in leafy vegetables by ultrasonic solvent extraction and liquid chromatographytandem mass spectrometry. Ultrasonics Sonochemistry 15(1): 25-32.

9. Masahiro O, Kitagawa Y, Akutsu K, Hirotaka O, Yukio T, et al. (2005) Rapid method for the determination of 180 pesticide residues in foods by gas chromatography/mass spectrometry and flame photometric detection. ] Pestic Sci 30(4): 368-377.

10. Zorka K, Serdar M (2009) Screening of fresh fruit and vegetables for pesticide residues on Croatian market. Food Control 20(4): 419-422.

11. Lin Juan Qu, Hui Zhang, Jian Hua Zhu, Guo Sheng Yang, Hassan Y Aboul Enein (2010) Rapid determination of organophosphorus pesticides in leeks by gas chromatography-triple quadrupole mass spectrometry. Food Chem 122(1): 327-332.

12. Mol H G, Van Dam R C, Steijger OM (2003) Determination of polar organophosphorus pesticides in vegetables and fruits using liquid chromatography with tandem mass spectrometry: selection of extraction solvent. J Chromatogr A 1015(1-2): 119-127.

13. Zheng HB, Zhao Q Mo J Z, Huang YQ, Luo YB, et al. (2013) Quick, easy, cheap, effective, rugged and safe method with magnetic graphitized carbon black and primary secondary amine as adsorbent and its application in pesticide residue analysis. J Chromatogr A 1300:127-133.

14. Christia C, Bizani E, Christophoridis C, Fytianos K (2015) Pesticide residues in fruit samples: comparison of different QuEChERS methods using liquid chromatography-tandem mass spectrometry. Environ Sci Pollut Res 22(17): 13167-13178.

15. Bemph CK, Augustine KD (2011) Pesticide residues in fruits at the market level in Accra Metropolis, Ghana, a preliminary study. Environ Monit Assess 175(1-4): 551-561.

ISSN: 2574-1241

DOI: $10.26717 /$ BJSTR.2020.24.004035

Salah Eldeen A Ali. Biomed J Sci \& Tech Res

CC (P) This work is licensed under Creative BY Commons Attribution 4.0 License

Submission Link: https://biomedres.us/submit-manuscript.php
16. Adalberto, Menezes Filho, SN, Haroldo (2006) SD Development of MSPD Method for the Determination of Pesticide Residues in Tomato by GCMS. Journal Brazilian Chemical Society 17(5): 874-879.

17. (2002) Commission Directive 2002/63/EC of 11 July 2002 establishing community methods of sampling for the official control of pesticide residues in and on products of plant and animal origin and repealing directive 79/700/EEC. Official Journal of European Communities L187: $30-43$.

18. Lehotay SJ, Son KA, Kwon H, Koesukwiwat U, Fu W, et al. (2010) Comparison of QuEChERS sample preparation methods for the analysis of pesticide residues in fruits and vegetables. J Chromatogr A 1217(16): 2548-2560.

19. (2008) FAO/WHO. Codex Pesticides Residue Online Database in/on Vegetables.

20. Bempah CK, Donkor A, Yeboah PO, Dubey B, Osei Fosu P (2011) A preliminary assessment of consumer's exposure to organochlorine pesticides in fruits and vegetables and the potential health risk in Accra Metropolis, Ghana. Food Chem 128(4): 1058-1065.

21. Osman KA, Al Humaid AI, Al Rehiayani SM, Al Redhaiman KN (2011) Estimated daily intake of pesticide residues exposure by vegetables grown in greenhouses in Al-Qassim region, Saudi Arabia. Food Control 22(6): 947-953

22. Qin G,Zou K, LiY, Chen Y, HeF, etal. (2016) Pesticide residue determination in vegetables from western China applying gas chromatography with mass spectrometry. Biomed Chromatogr 30(9): 1430-1440.

23. Magdoleen A A (2002) Seasonal variation of pesticides residues in some salad vegetables in Khartoum State- Sudan. University of Khartoum.

24. Safiqul I, Mohammad D, Khabir U (2019) Determination of Major Organophosphorus Pesticide Residues in Eggplant using QuEChERS Extraction and Gas Chromatography. International Journal of Innovative Science and Research Technology 4(8): 212-219.

25. Gang W, Ming M, Hua Z, Rong B, YR Bian (2005) Organophosphorus pesticide extraction and cleanup from soils and measurement using GCNPD. Pedosphere 15(3): 386-394.

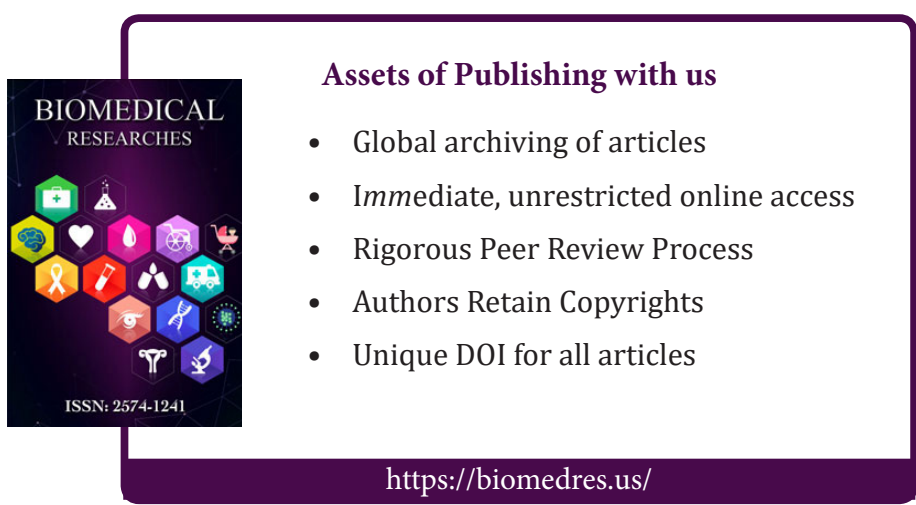

